\title{
Correction to: Verifying System-Wide Properties of Industrial Component-Based Software
}

Thomas Neele, Marijn Rol, and Jan Friso Groote

\section{Correction to: \\ Chapter "Verifying System-Wide Properties of Industrial Component-Based Software" in: H. Hojjat and M. Massink (Eds.): Fundamentals of Software Engineering, LNCS 11761, https://doi.org/10.1007/978-3-030-31517-7_11}

In Definition 2 of the originally published version, the first equation and the second paragraph were missing and the block of 5 equations was not aligned correctly. This has been corrected. 